\title{
Comparison of Olecranon Osteotomy with an Olecranon Sparing Technique for Fixation of Geriatric Intra-Articular Distal Humerus Fractures: The Enhanced Bag of Bones
}

Philip B. Kaiser, M.D., Erik T. Newman, M.D., Christopher Haggerty, B.A., Paul T. Appleton, M.D., John J. Wixted, M.D., Edward K. Rodriguez, M.D.

\section{Introduction}

Intra-articular distal humerus fractures (IADHFs) are disabling injuries and difficult to treat in the elderly. Limited open reduction internal fixation (ORIF) without olecranon osteotomy (OcO) or anatomic joint reduction and fixation referred to as the "enhanced bag of bones" (EBoB) hasn't been formally compared to the more traditional ORIF in this population.

\section{Objective}

The objective of this study was to compare pain scores and clinical outcome in elderly patients treated with ORIF+ OcO compared to those who underwent the EBoB technique.

\section{Methods}

56 patients ( $\geq 65$ years old) with IADHFs, classified as AO humeral 13-C type fractures, who underwent surgical fixation between July 1, 2005 and July 1, 2017 and with at least 12 months of follow-up were retrospectively reviewed.

\section{ORTHOPEDIC TRAUMA} INITIATIVE HARVARD MEDICAL SCHOOL

\section{Methods (Cont.)}

The primary outcomes of this study were final elbow range of motion (in the coronal plane), complications, and the need for additional elbow surgery. Secondary outcomes included patientreported outcome measures for pain and function.

\section{Results}

Out of the 56 patients, 30 were treated with ORIF + OcO and 26 patients were treated with the EBoB technique. The mean follow-up duration for the study was 15.2 months (range 12-97 months). No statistically significant difference was found in the range of motion between the treatment groups.

There was a trend towards more complications and additional surgery in the ORIF+OcO group.

The average operative time was 168 minutes in the ORIF $+\mathrm{OcO}$ and 138 minutes in the EBoB group ( $p=0.04)$. PROMIS scores for pain and PROMIS function scores were very close in both groups.

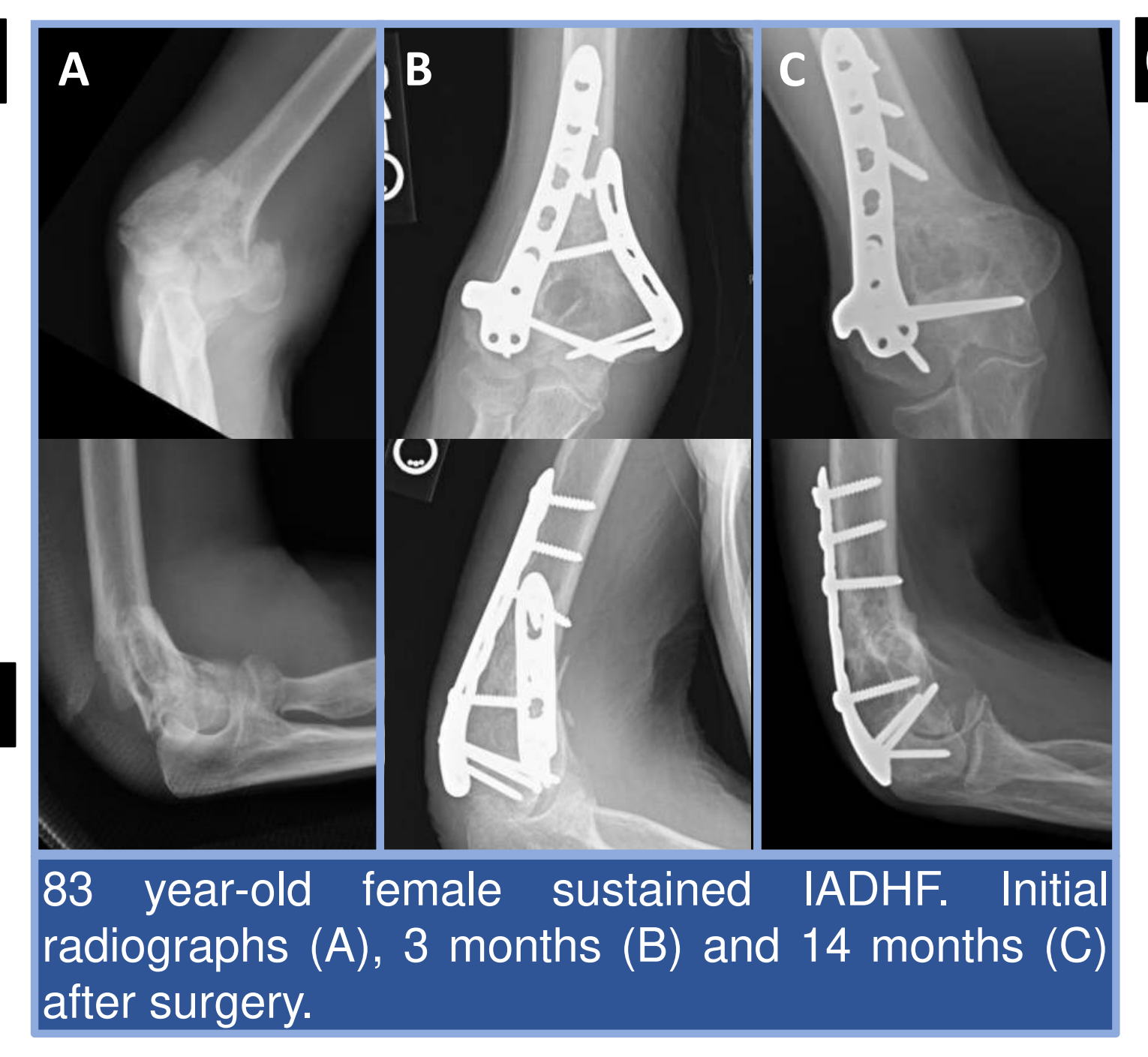

\section{Conclusions}

Treatment with the EBoB technique demonstrated equivalent outcomes with regards to $\mathrm{ROM}$, function, and pain in elderly patients with IADHFs when compared to the traditional ORIF+ OcO.

Surgical times were longer, and more complications and need for additional surgery were observed with ORIF+OcO.

The EBoB technique should be considered in the treatment algorithm of certain elderly patients with IADHFs.

\begin{tabular}{|l|ccc|}
\hline \multicolumn{1}{|c|}{ Outcomes for Treatment Groups } & ORIF+OcO $(\mathrm{N}=30)$ & EBoB $(\mathrm{N}=26)$ & $p$-vlaue \\
\cline { 1 - 2 } Final Extension (degrees short of $\left.0^{\circ}\right)$ & $22.5^{\circ}\left(0^{\circ}-45^{\circ}\right)$ & $26.9^{\circ}\left(0^{\circ}-90^{\circ}\right)$ & 0.53 \\
\cline { 1 - 1 } Final flexion & $119.5^{\circ}\left(90^{\circ}-150^{\circ}\right)$ & $113.4^{\circ}\left(90^{\circ}-150^{\circ}\right)$ & 0.3 \\
\cline { 1 - 1 } ROM & $97^{\circ}\left(40^{\circ}-155^{\circ}\right)$ & $86.5^{\circ}\left(20^{\circ}-145^{\circ}\right)$ & 0.23 \\
\cline { 1 - 1 } Complications & $11(34 \%)$ & $4(15 \%)$ & 0.07 \\
\cline { 1 - 1 } Additional surgery & $10(31 \%)$ & $4(15 \%)$ & 0.12 \\
\cline { 1 - 1 } PROMIS Pain & 53.1 & 52.14 & 0.86 \\
\cline { 1 - 1 } PROMIS Function & 41.7 & 41.4 & 0.95 \\
\hline
\end{tabular}

